\title{
Ultrahigh Strength Single Crystalline Nanowhiskers Grown by Physical Vapor Deposition
}

\author{
Gunther Richter, ${ }^{*, \dagger}$ Karla Hillerich, ${ }^{\ddagger \perp}$ Daniel S. Gianola, ${ }^{\S}$ Reiner Mönig,§ \\ Oliver Kraft, ${ }^{\S}$ and Cynthia A. Volkert" \\ Max-Planck-Institut für Metallforschung, Heisenbergstrasse 3, \\ D-70589 Stuttgart, Germany, Institut für Metallkunde, Universität Stuttgart, \\ Heisenbergstrasse 3, D-70589 Stuttgart, Germany, Institut für Materialforschung II, \\ Forschungszentrum Karlsruhe, Hermann-von-Helmholtz-Platz 1, \\ D-76344 Eggenstein-Leopoldshafen, Germany, and Institut für Materialphysik, \\ Georg-August Universität Göttingen, Friedrich-Hund-Platz 1, D-37077 Göttingen
}

Received May 12, 2009; Revised Manuscript Received July 8, 2009

\begin{abstract}
The strength of metal crystals is reduced below the theoretical value by the presence of dislocations or by flaws that allow easy nucleation of dislocations. A straightforward method to minimize the number of defects and flaws and to presumably increase its strength is to increase the crystal quality or to reduce the crystal size. Here, we describe the successful fabrication of high aspect ratio nanowhiskers from a variety of face-centered cubic metals using a high temperature molecular beam epitaxy method. The presence of atomically smooth, faceted surfaces and absence of dislocations is confirmed using transmission electron microscopy investigations. Tensile tests performed in situ in a focusedion beam scanning electron microscope on Cu nanowhiskers reveal strengths close to the theoretical upper limit and confirm that the properties of nanomaterials can be engineered by controlling defect and flaw densities.
\end{abstract}

Single crystalline metal wires, or metal whiskers, with diameters larger than one micrometer have been routinely fabricated ${ }^{1}$ and used for experimentally examining mechanical, ${ }^{2}$ ferromagnetic, ${ }^{3}$ superconductive, ${ }^{4}$ and electronic ${ }^{5}$ properties. Interest in metal whiskers became intense once it was observed that they could exhibit strengths close to theoretical (ideal) values. ${ }^{2,6}$ Whiskers have been grown by the vapor liquid solid method $^{7}$ (VLS) or metal halide reduction, ${ }^{6}$ the latter of which have demonstrated high strength. ${ }^{6}$ This has been attributed to the near-equilibrium nature of the growth process and the resultant absence of defects and flaws in the samples. Extending the fabrication of high quality metal whiskers to submicrometer diameters, as routinely produced for semiconductors, ${ }^{7-9}$ is clearly desired but has been largely elusive. However, single crystalline metal nanowires have only occasionally been successfully fabricated. ${ }^{10}$ Here we describe the first time to our knowledge the fabrication of metal single crystalline nanowhiskers (NWs) with diameters

\footnotetext{
* To whom correspondence should be addressed. E-mail: Richter@ mf.mpg.de.

${ }^{\dagger}$ Max-Planck-Institut für Metallforschung.

* Universität Stuttgart.

$\S^{\S}$ Forschungszentrum Karlsruhe.

"Georg-August Universität Göttingen.

${ }^{\perp}$ Present Address: Solid State Physics, Lund University, Box 118, S-22100 Lund, Sweden.
}

as small as $20 \mathrm{~nm}$ and no defects, as evidenced by their strengths near the theoretical upper limit. Such nanostructures have the potential to serve as model systems for elucidating intrinsic properties in tiny structures, such as quantum effects, and to be used as building blocks in nanotechnological applications where unique functionalities are required.

In this study, free-standing, high aspect ratio, single crystalline nanowhiskers of a variety of different materials (copper, gold, silver, aluminum, and silicon) have been successfully grown from partially C-coated, oxidized and nonoxidized Si (100), (110), and (111) substrates under molecular beam epitaxy (MBE) conditions. Both elevated substrate temperatures (on the order of $0.65 T_{\mathrm{M}}$ of the deposited species) and the partial $\mathrm{C}$ layer are necessary to achieve nanowhisker growth. In the remainder of this publication we will focus on results from $\mathrm{Cu}$ nanowhiskers.

Figure 1 shows scanning electron micrographs of two $\mathrm{Cu}$ samples with nominal $\mathrm{Cu}$ thicknesses of 45 and $200 \mathrm{~nm}$, respectively. In addition to whiskers, which are straight and constant in diameter over their length, $\mathrm{Cu}$ islands, platelets, and silicide ridges have formed on the substrate. It was observed that whisker growth strongly depends on the carbon layer. Whiskers could not be found on samples without $\mathrm{C}$ layer and also did not grow on samples where the $\mathrm{C}$ layer 

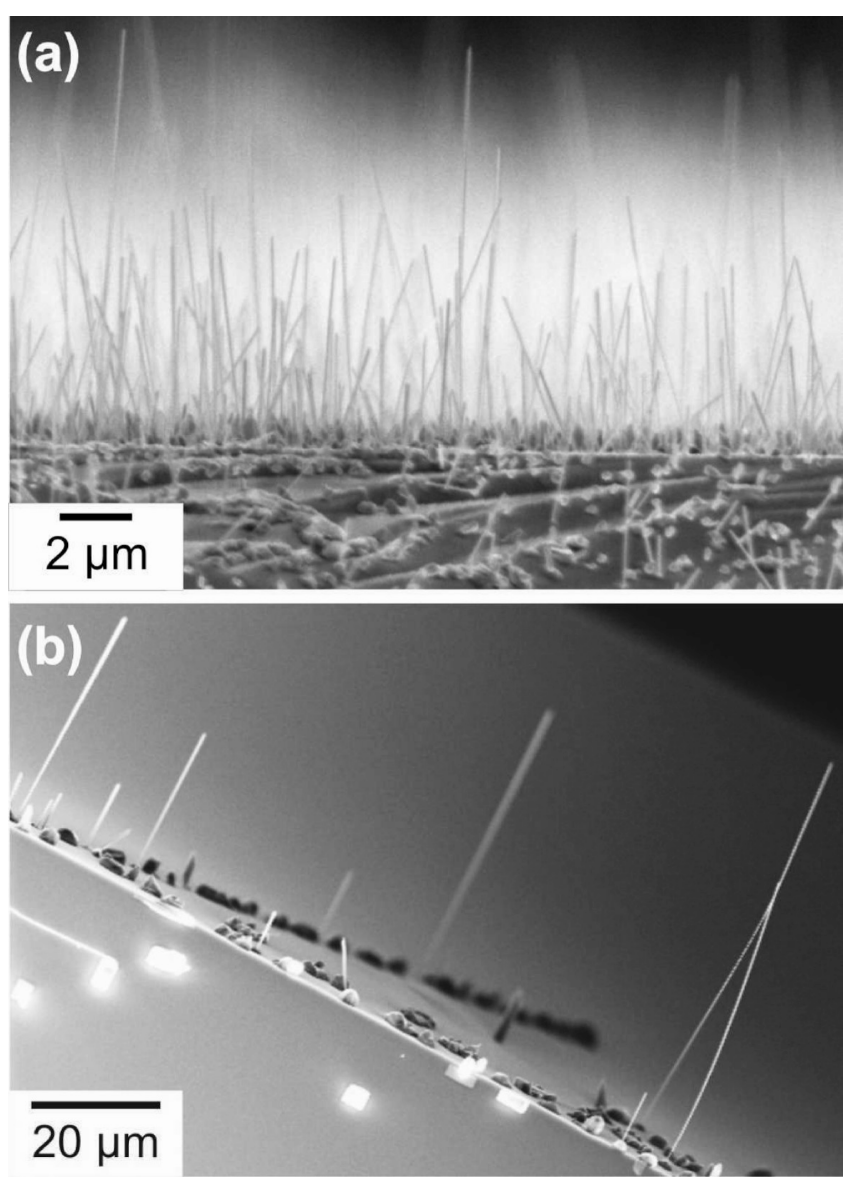

Figure 1. SEM images of $\mathrm{Cu}$ whiskers. (a) Nominal film thicknesses of $45 \mathrm{~nm} \mathrm{Cu}$ were thermally evaporation deposited at 650 ${ }^{\circ} \mathrm{C}$ with a nominal deposition rate of $R=0.05 \mathrm{~nm} / \mathrm{s}$. The NWs are straight with a maximum angle of $30^{\circ}$ of to the surface normal. The nanowhiskers shown in this micrograph have a maximum length of $10 \mathrm{um}$, and the diameters vary from 30 to $100 \mathrm{~nm}$. However, whiskers have been grown with diameters as small as $20 \mathrm{~nm}$ and as large as $250 \mathrm{~nm}$, and with lengths as high as 320 $\mu \mathrm{m}$. (b) NWs formed on one of the cleavage planes. The projected length is $75 \mu \mathrm{m}$ and the diameter is $150 \mathrm{~nm}$. The whiskers are free-standing and appear perpendicular to the surface

was thicker than $40 \mathrm{~nm}$. Although the effect of different carbon layer thicknesses was investigated, no correlation between the whisker dimensions and $\mathrm{C}$ layer thickness could be identified. The carbon mediated whisker growth is consistent with earlier observations of whisker growth from several decades ago. ${ }^{11}$ Although a halide process was used for the growth, it was found that whisker growth is suppressed on flat and clean substrates. Microscopic observations of the nanowhiskers show that they have a strong tendency to form directly on the oxidized $\mathrm{Si}$ wafer at openings in the $\mathrm{C}$ film (Supporting Information, Figure $\mathrm{S} 1$ ). From scanning electron microscopy (SEM) images, it was found that the axis of the majority of the whiskers has an angle of less than $30^{\circ}$ to the substrate normal. Although the whiskers show no taper, they grow in length and diameter during deposition. This was qualitatively confirmed by experiments where different amounts of $\mathrm{Cu}$ were deposited and the dimensions of the resulting whiskers were observed using SEM. In the particular example of Figure 1a, the whiskers exhibit a wide distribution of diameters ranging from 20 to $100 \mathrm{~nm}$ and an average length of approximately $10 \mu \mathrm{m}$, giving aspect ratios as large as 300 . Larger nominal $\mathrm{Cu}$ film thicknesses produce larger whisker lengths and diameters up to $300 \mathrm{~nm}$ in diameter and $75 \mu \mathrm{m}$ in length (Figure 1b).

A representative dark-field transmission electron microscopy (TEM) image of a $200 \mathrm{~nm}$ diameter $\mathrm{Cu}$ whisker is shown in Figure 2a. The whisker axis is parallel to the [01] crystallographic direction, as determined by selected area electron diffraction. $\langle 110\rangle$ oriented whiskers are observed to be dominant for all whisker diameters. This is in accordance with the results from vapor grown whiskers observed by in situ field ion microscopy. ${ }^{12}$ The dark-field image (Figure 2a) was taken under a two beam condition with a diffraction vector of $g=(\overline{1} 1 \overline{1})$ and shows no dislocations. Images for two additional diffraction vectors (Supporting Information, Figure S2) and the invisibility criterion rule out all dislocations except those with burgers vectors parallel to the [011] direction, which explicitly eliminates the possibility of partial dislocations or a screw dislocation along the whisker axis. In addition, the smooth contrast observed under all beam conditions and at all angles suggests the absence of any $<011>$ burger vector dislocations as well as the absence of a thick surface oxide. Similar results from microscopy investigations of other nanowhiskers of $\mathrm{Cu}$ and other materials (Ag, $\mathrm{Au}, \mathrm{Al}, \mathrm{Si}$ ) show that the whiskers investigated are also dislocation-free.

Energy filtered TEM was used to determine the crosssectional shape of the whiskers and to rule out extensive contamination. The uniformity of the $\mathrm{Cu}$ intensity in the nanowire images (Supporting Information, Figure S3) indicates the absence of thick surface oxides or growth catalyst particles. Thickness maps derived from electron energy loss spectroscopy (EELS) measurements for a $75 \mathrm{~nm}$ diameter whisker are shown in Figure 2b,c. Integrated profiles of the image intensities are shown with the projected mass thicknesses shown as insets. The specimen was rotated between images by $30^{\circ}$ around the whisker axis. The different profiles clearly indicate faceting of the nanowhisker. All TEM micrographs (Figure $2 \mathrm{a}-\mathrm{c}$ and Supporting Information, Figure S2) suggest faceting of the ends of the nanowhiskers as well with a typical included angle of approximately $70^{\circ}$.

The faceted shapes of the nanowhiskers are in accordance with previous observations of macrowhisker growth ${ }^{6,13,14}$ and suggest that surface energy plays an important role in whisker formation and growth. The cross-sectional shape for a $\mathrm{Cu}$ whisker with a $[01 \overline{1}]$ axis, calculated using the Wulff construction and literature values for $\mathrm{Cu}$ surface energies $\left(\gamma_{\{100\}} / \gamma_{\{111\}}=1.30, \gamma_{\{110\}} / \gamma_{\{111\}}=1.35\right),{ }^{15}$ is shown in the inset of Figure 2d. The integrated thickness taken along two different directions (I and II in Figure 2d) are shown as dashed lines in the insets of Figure 2b,c and agree very well with the experimental profiles. The nanowhisker tip facets can be calculated analytically after Wulff by fixing the whisker cross-sectional shape and minimizing the surface energy while varying the tip facet area, resulting in the formation of $\{111\}$ and $\{100\}$ crystal planes. A shape model can be derived from these calculations and is presented in 

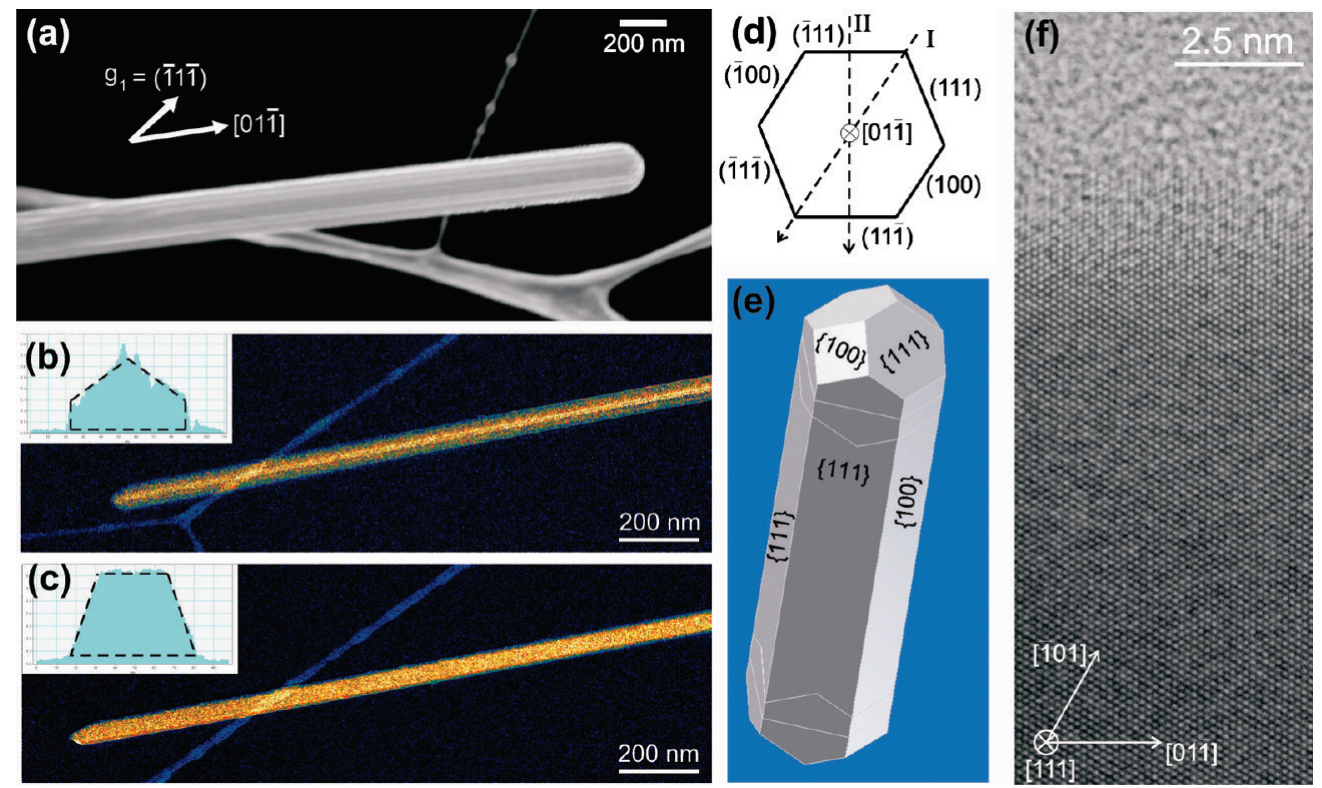

Figure 2. Morphology of $\mathrm{Cu}$ whisker. (a) Dark-field TEM image taken in a two beam condition with diffraction vector $\mathbf{g}_{1}=(\overline{1} 1 \overline{1})$. The growth direction of the whisker is [011]. No dislocations are visible, particularly no screw dislocations parallel to the whisker centerline are observed. (b,c) Energy filtered TEM micrographs. (c) The whisker shown in (b) is rotated by $30^{\circ}$ along the whisker axis. The corresponding projected thickness profile is drawn in the inset, and the dashed lines indicate projected thickness from Wulff calculations. (d) Ideal whisker shape calculated by $\mathrm{Cu}$-free surface energies using the Wulff plot, as shown projected in the [011] direction. The projection directions for $(\mathrm{b}, \mathrm{c})$ are labeled I and II, respectively. (e) $\mathrm{Cu}$ whisker shape model based on Wulff plot, where the whisker side- and tip-facets are formed by $\{111\}$ and $\{100\}$ crystal planes. (f) High resolution TEM in [111] zone axis. No structural contamination is detectable either on the surface or in the bulk.

Figure 2e. The tip facet angle of $70^{\circ}$ determined experimentally from TEM images corroborates with the geometry of two intersecting $\{111\}$ facets that dominate the tip shape. The well-defined facets suggest that the growth is a nearequilibrium process. Figure $2 \mathrm{f}$ shows a high-resolution TEM phase contrast image of a whisker. No structural contaminations on the surface or in the bulk of the nanowhisker are detectable. A thin layer presumably consisting of $\mathrm{CuO}$ was found which most likely formed on the whisker surface after removal from the vacuum system. Especially no ordered C contamination can be seen on the surface

The results shown so far for the $\mathrm{Cu}$ nanowhiskers are representative for all materials. Similar high aspect ratios, single crystalline growth was also achieved for $\mathrm{Ag}, \mathrm{Au}, \mathrm{Al}$, and $\mathrm{Si}$, (Supporting Information, Figure S4). Distributions in density, diameter, and length varied from material to material and may originate from the nanowhisker formation and growth process itself. Various growth models have been proposed for the formation of whiskers. The oldest model is based on the Frank-Cabrera mechanism for crystal growth, where atoms are added at the step edge produced by a threading screw dislocation and requires the screw dislocation to extend along the whisker axis. ${ }^{16,17}$ Since no dislocations, particularly screw dislocations parallel to the whisker axis, are observed in our TEM studies, we rule out this possibility. A second concept for whisker formation, the vapor-liquidsolid or VLS model, ${ }^{7}$ has been used to explain catalyzed whisker growth. Unless the catalyst is used up during the growth, one expects to find it at the whisker tip or root. No catalyst particle remains at the whisker tip of the samples grown here.
Two processes must occur for whisker formation: first, nucleation has to take place, and subsequently axial growth at the expense of radial growth. In the case here where $\mathrm{C}$ is essential to the whisker formation process, $\mathrm{C}$ must play a role in one or both steps. The effect of $\mathrm{C}$ morphology on $\mathrm{Cu}$ diffusion is well known ${ }^{18-20}$ and will surely influence the location and density of whisker nuclei. One can envision a process whereby deposited $\mathrm{Cu}$ atoms diffuse across the $\mathrm{C}$ surface to nucleate islands or clusters in openings in the $\mathrm{C}$ film. Since the interfacial energy between $\mathrm{Cu}$ and $\mathrm{C}$ is high, ${ }^{21}$ it is energetically unfavorable for nuclei to grow laterally onto the $\mathrm{C}$ film. This could help to force the nuclei to grow vertically. Once nuclei have formed, further growth occurs by diffusion of $\mathrm{Cu}$ adatoms on the $\mathrm{C}$ covered substrate to the existing nuclei and incorporation. Except in late stages of growth, the flux of $\mathrm{Cu}$ atoms impinging directly on the whisker can be neglected relative to that diffusing on the $\mathrm{C}$ covered substrate. ${ }^{22}$ The fact that no tapering was observed, even for very high aspect ratio whiskers, indicates that diffusion on the side facets must be rapid relative to incorporation (so-called reaction-limited growth). Even at low super saturations some nuclei form on the whisker side facets increasing the whisker diameter with time.

This all suggests that the key to the whisker growth is some highly preferred site for $\mathrm{Cu}$ incorporation, most likely at the whisker root. ${ }^{23}$ Although the exact microstructure of this site is not yet identified, its high selectivity is presumably responsible for the high crystal quality and faceted surfaces obtained during growth.

Low densities of defects or surface irregularities are difficult to directly quantify in metal nanowhiskers and it is 

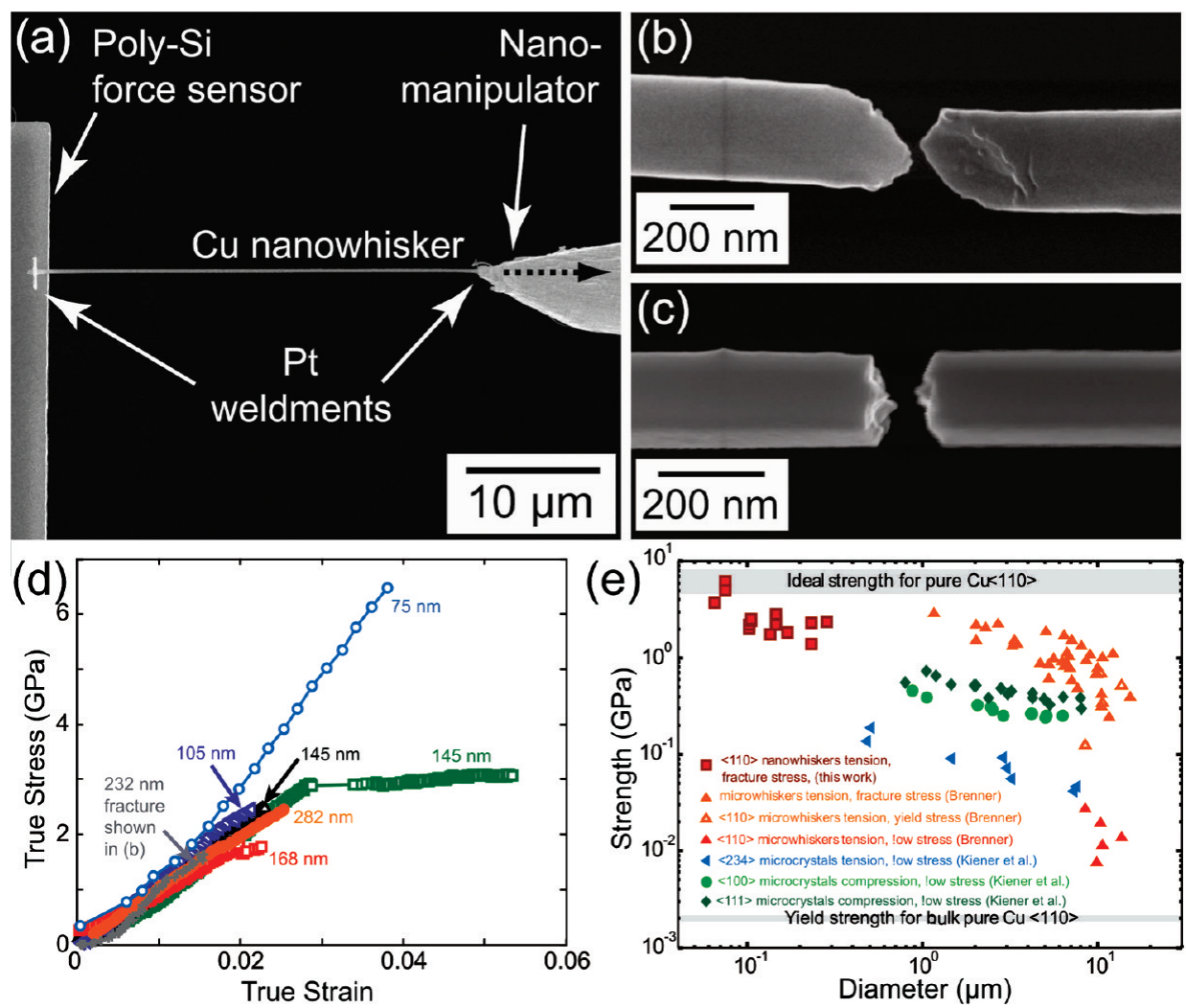

Figure 3. Tensile testing of individual Cu whiskers (a) SEM micrograph of whisker mechanical testing configuration. Whiskers are gripped by e-beam deposited Pt weldments, the force applied on the specimen is measured by either a poly-Si flexure beam apparatus or a capacitivebased transducer, and the local strain is measured by digital image correlation of SEM micrographs obtained during testing. (b,c) SEM micrographs of fracture surfaces of deformed whiskers showing shear (b) and brittle (c) failure. (d) Representative stress-strain curves for $\mathrm{Cu}$ whiskers of varying size. (e) Strength plotted as a function of whisker diameter and compared to reported values of strength of pure $\mathrm{Cu}$ single crystalline small scale specimens. $2,33,36,37$

expected that mechanical strength is a more stringent metric for crystal and surface quality than most structural characterization methods. To perform mechanical tests, individual $\mathrm{Cu}$ whiskers with diameters between 75 and $300 \mathrm{~nm}$ were selected from the substrate and mounted in a tensile testing setup inside a dual beam SEM/FIB using an in situ nanomanipulator. Quantitative tensile tests were performed during SEM observation (Figure 3a), and force and displacement were measured and used to calculate stress and strain. The stress-strain behavior was found to be more or less linear until apparent brittle fracture occurred. Small deviations from linearity were sometimes measured and could be attributed to either straightening of the nanowire during initial loading (positive curvature, e.g., $75 \mathrm{~nm}$ diameter nanowire) or small amounts of inelastic deformation (negative curvature, e.g., $168 \mathrm{~nm}$ diameter nanowire). A more pronounced inelastic deformation was observed in only one case, but the specimen was not able to be transferred for postmortem characterization to investigate this anomalous response. In thicker wires $(>200 \mathrm{~nm})$, fracture sometimes took place along a plane or planes with orientations relative to the tensile axis consistent with the $\{111\}$ plane with the highest resolved shear stresses (Figure 3b). This suggests that the apparent fracture may be due to highly localized dislocation activity or shear failure at the theoretical strength. In most other cases, fracture surfaces appeared rough and were generally oriented perpendicular to the tensile axis (Figure 3c) indicating a purely brittle fracture mode in the absence of dislocations. Regardless of the fracture morphology, no plastic necking was observed in any of the experiments.

Apparent brittle fracture occurred at stresses lying between 1 and 7 GPa (Figure 3d). These fracture strengths are well in excess of the yield stress of high purity bulk single crystalline $\mathrm{Cu}$ ( $>1000$ times higher) and still higher than the yield strengths measured in $\mathrm{Cu}$ microwhiskers. ${ }^{2,6}$ Calculations of the resolved shear stress at fracture on the highest Schmid factor slip systems result in stresses that are consistent with calculated theoretical shear strengths of pure $\mathrm{Cu}^{24-26}$ Normalized shear strengths range from $\mu / 50$ to $\mu / 11$, where $\mu$ is the shear modulus of $\mathrm{Cu}$ along $<110\rangle$ directions. Fracture strengths for a range of sizes showed significant scatter (Figure 3e), which could be interpreted in the context of a weakest link framework, as often employed in the fracture of ceramics and glasses. ${ }^{27}$ Indeed, this explanation was employed by Brenner to describe the high yield strength of ductile metal microwhiskers. ${ }^{2}$ It also appears to fit the experiments here, particularly given the absence of dislocations observed by TEM in the whiskers. The increased strengths of the nanowhiskers relative to the microwhiskers ${ }^{2,6}$ and large scatter in strengths is rationalized by the small volumes exposed to tensile stresses and the lowered probability of encountering critical defects in these whiskers.

It is clear that large-scale plasticity is not sustainable in these whiskers, as supported by the lack of plastic flow in 
the stress strain curves, as well as postmortem TEM characterization showing an absence of stored dislocations in fractured whiskers. These results are quite different from the results obtained from a series of small-scale experiments on face-centered cubic (fcc) single crystals of arguably lower crystal quality, which demonstrate smaller strengths with less scatter, and a clear size effect ("smaller is stronger"). ${ }^{28-33}$ This behavior has been attributed to constrained dislocation interactions and geometrical limitations of source size for samples with dimensions between around $200 \mathrm{~nm}$ and 10 $\mu \mathrm{m}$. For smaller samples, it is observed that dislocations run out of the samples, ${ }^{34,35}$ leaving dislocation nucleation the only possible process for controlling further deformation. Upon nucleating dislocations in these nanowhiskers with good crystal quality and thus few critical defects, the deformation is expected to occur heterogeneously, which is commensurate with the absence of tensile necking. Slip will be localized to one single crystal plane since no other flaws are in the vicinity that can trigger subsequent plastic deformation by this activity. Presumably the strength of these small samples is controlled by the strength of the flaws for nucleating new dislocations and will depend critically on the sample preparation method.

We report a novel synthesis route for producing single crystalline, high-aspect ratio, defect-free nanostructures of a variety of pure materials. A combination of electron microscopy techniques show that $\mathrm{Cu}$ nanowhiskers have no detectable defects and crystal shapes that bear resemblance to those formed during equilibrium growth. Novel and directly interpretable in situ mechanical experiments reveal ultrahigh strengths, which to our knowledge, are the highest ever reported in $\mathrm{Cu}$. The absence of large amounts of plasticity, in contrast to that typically encountered in pure fcc metals, and the very high mechanical strength are attributed to the small sizes and crystalline quality of these nanowhiskers. Both factors are likely to influence other physical properties such as electrical and thermal conductivity. An understanding of the fabrication and structural conditions required to achieve such metal structures will have implications on the development of high performance materials for nanotechnological applications.

Acknowledgment. K.H. and G.R. gratefully acknowledge Mr. Michael Pudleiner for carrying out the thermal evaporation of the materials and Mr. Reinhart Völker for magnetron sputtering of the $\mathrm{C}$ layers. Mr. Kersten Hahn is acknowledged for the operation of the energy filtered-TEM. They are also much obliged to Professor Eduard Arzt for the possibility that part of this work was carried out as a master thesis in his departments at the MPI für Metallforschung and the
University of Stuttgart. D.S.G. acknowledges support from an Alexander von Humboldt Postdoctoral Fellowship.

Supporting Information Available: Experimental methods (materials deposition, microstructure characterization, in situ tensile testing) and additional figures (nanowhiskers from other materials, further microstructure characterizations). This material is available free of charge via the Internet at http:// pubs.acs.org.

\section{References}

(1) Hardy, H. K. Prog. Met. Phys. 1956, 6, 45-73.

(2) Brenner, S. S. J. Appl. Phys. 1956, 27, 1484-1491.

(3) DeBlois, R. W.; Graham, C. D. J. Appl. Phys. 1958, 29, 931-939.

(4) Lutes, O. S. Phys. Rev. 1957, 105, 1451-1458.

(5) Shoenberg, D. Nature 1959, 183, 171.

(6) Brenner, S. S. Acta Met. 1956, 4, 62-74.

(7) Wagner, R. S.; Ellis, W. C. Appl. Phys. Lett. 1964, 4, 89.

(8) Duan, X.; Huang, Y.; Cui, Y.; Wang, J.; Lieber, C. M. Nature 2001, 409, 66-69.

(9) Borgström, M.; Deppert, K.; Seifert, W. J. Cryst. Growth 2004, 260, $18-22$.

(10) Karim, S.; et al. Appl. Phys. A 2006, 84, 403-407.

(11) Gomer, R. In Growth and Perfection of Crystals; Doremus, R. H., Roberts, B. W., Turnbull, D., Eds.; Wiley: New York, 1958; pp 126133.

(12) Melmed, A. J.; Gomer, R. J. Chem. Phys. 1961, 34, 1802-1812.

(13) Brenner, S. S.; Morelock, C. R. Rev. Sci. Instrum. 1957, 28, 652653.

(14) Nittono, O.; Nagakura, S. Jpn. J. Appl. Phys. 1969, 8, 1180-1188.

(15) Rodríguez, A. M.; Bozzolo, G.; Ferrante, J. Surf. Sci. 1993, 289, 100126.

(16) Sears, G. W. Acta Met. 1955, 3, 361-369.

(17) Frank, F. C. Disc. Faraday Soc. 1949, 5, 48-54.

(18) Wang, P. I.; Zhao, Y. P.; Wang, T. M.; Lu, T. M. Nanotechnology 2004, 15, 218-222.

(19) Egelhoff, W. F.; Tibbetts, G. G. Phys. Rev. B 1979, 19, 5028-5035.

(20) Hamilton, J. F.; Logel, P. C. Thin Solid Films 1973, 16, 49-63.

(21) Mortimer, D. A.; Nicholas, M. J. Mater. Sci. 1970, 5, 149-155.

(22) Ruth, V.; Hirth, J. P. J. Chem. Phys. 1964, 41, 3139-3149.

(23) Inkson, B. J.; Dehm, G.; Peng, Y. Nanotechnology 2007, 18, 1-4.

(24) Ogata, S.; Li, J.; Yip, S. Science 2002, 298, 807-811.

(25) Roundy, D.; Krenn, C. R.; Marvin, L.; Cohen, M. L.; Morris, J. W., Jr. Phys. Rev. Lett. 1999, 82, 2713-2716.

(26) Paxton, A. T.; Gumbsch, P.; Methfessel, M. Philos. Mag. Lett. 1991, 63, 267-274.

(27) Weibull, W. Proc. R. Swedish Inst. Eng. Res. 1939, 151, 1-45.

(28) Uchic, M. D.; Dimiduk, D. M.; Florando, J. N.; Nix, W. D. Science 2004, 305, 986-989.

(29) Greer, J. R.; Oliver, W. C.; Nix, W. D. Acta Mater. 2005, 53, 18211830.

(30) Greer, J. R.; Oliver, W. C.; Nix, W. D. Acta Mater. 2006, 54, 1705.

(31) Volkert, C. A.; Lilleodden, E. T. Philos. Mag. 2006, 86, 5567-5579.

(32) Gruber, P. A.; Solenthaler, C.; Arzt, E.; Spolenak, R. Acta Mater. 2008, 56, 1876-1889.

(33) Kiener, D.; Grosinger, W.; Dehm, G.; Pippan, R. Acta Mater. 2008, 56, 580-592.

(34) Shan, Z. W.; Mishra, R. K.; Asif, S. A.; Warren, O. L.; Minor, A. M. Nat. Mater. 2008, 7, 115-119.

(35) Oh, S. H.; Legros, M.; Kiener, D.; Dehm, G. Nat. Mater. 2009, 8, 95-100.

(36) Brenner, S. S. J. Appl. Phys. 1957, 28, 1023-1026.

(37) Kiener, D.; Motz, C.; Schoberl, T.; Jenko, M.; Dehm, G. Adv. Eng. Mater. 2006, 8, 1119-1125.

NL9015107 